\title{
eJRIEPS
}

Ejournal de la recherche sur l'intervention en éducation physique et sport

$20 \mid 2010$

Varia

\section{Représentations sociales de la lutte sénégalaise : perspectives d'élaboration de contenus}

Cheikh Tidiane Wane, Laboratoire VST2I, Université Bordeaux 2, EA 4140 ** Laboratoire Circeft, Université Paris Est Créteil, E.A. 4384 et JeanFrançois Robin

\section{OpenEdition}

Journals

Édition électronique

URL : http://journals.openedition.org/ejrieps/4864

DOI : $10.4000 /$ ejrieps.4864

ISSN : 2105-0821

Éditeur

ELLIADD

\section{Référence électronique}

Cheikh Tidiane Wane, Laboratoire VST2I, Université Bordeaux 2, EA 4140 ** Laboratoire Circeft, Université Paris Est Créteil, E.A. 4384 et Jean-François Robin, « Représentations sociales de la lutte sénégalaise : perspectives d'élaboration de contenus », eJRIEPS [En ligne], 20 | 2010, mis en ligne le 01 avril 2010, consulté le 09 avril 2020. URL : http://journals.openedition.org/ejrieps/4864 ; DOI : https:// doi.org/10.4000/ejrieps.4864

Ce document a été généré automatiquement le 9 avril 2020.

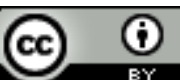

La revue eJRIEPS est mise à disposition selon les termes de la Creative Commons Attribution 4.0 International License. 


\title{
Représentations sociales de la lutte sénégalaise : perspectives d'élaboration de contenus
}

\author{
Cheikh Tidiane Wane, Laboratoire VST2I, Université Bordeaux 2, EA 4140 \\ ** Laboratoire Circeft, Université Paris Est Créteil, E.A. 4384 et Jean- \\ François Robin
}

1 D'une façon générale, la lutte est une pratique populaire dans de nombreux pays. Elle est certainement une des disciplines individuelles les plus pratiquées dans le monde (Clément \& Lacaze, 1985). À la fois pratique sportive et artistique, la lutte se pratique sous une forme spontanée chez les enfants. Dans certaines sociétés (notamment dans les pays de l'Europe de l'est, en Égypte, en Turquie ou au Sénégal), elle revêt un caractère prestigieux. Lutter, c'est l'art de faire passer les émotions et les actions dans l'âme du spectateur par l'expression vraie des mouvements, des gestes, du corps et de la culture. La lutte au Sénégal s'inscrit dans le processus de passage des jeux aux sports selon le phénomène de sportivisation des jeux traditionnels, identifié par During (1984) et Bourdieu (1984). Elle apparaît comme une pratique sportive à part entière. Elle reste une pratique sportive traditionnelle issue des traditions culturelles sénégalaises. En effet, la lutte demeure ancrée dans l'imaginaire sénégalais, lié au monde des croyances et des superstitions. Selon N'diaye (1996, p. 109), «à la fois sport, jeu et espace de création littéraire, où la poésie s'auréole de musique et s'innerve de rythme, la lutte est un phénomène immense au Sénégal. La place qui lui échoit se perçoit dans l'aménagement de l'espace habité où des aires de jeux multifonctionnels lui sont consacrées ». Contrairement à d'autres manifestations sociales sélectives relativement aux âges, aux sexes ou aux catégories socioprofessionnelles, la lutte constitue avec les joutes poétiques, l'une des rares manifestations qui convie l'ensemble du corps social à être témoin des prouesses de quiconque à la prétention de se présenter comme un champion. Nous nous demandons dans cette étude, si cette Activité Physique Sportive et Artistique (APSA) peut constituer une «pratique sociale de référence » (Martinand, 1994) suffisamment puissante enseignable à l'école. 
Autrement dit, il s'agit de déterminer quelle forme de lutte scolaire on peut proposer à des élèves compte tenu d'une part des contraintes de l'EPS au Sénégal et d'autre part de la signification culturelle et sociale de la lutte sénégalaise ? Nous nous inscrivons dans une approche anthropo-techno-didactique, que nous développerons selon deux axes dans cet article :

Un axe socio-historique, culturel et économique relatif au contexte et à l'activité support. Il s'agit de mieux caractériser l'activité (la lutte sénégalaise) afin d'extraire les éléments pouvant nous servir de référence.

Un axe technologique, qui nous permettra de modéliser l'activité de lutte scolaire.

\section{Cadre théorique}

2 Le cadre conceptuel de la recherche est construit autour de l'idée principale selon laquelle accéder à la compréhension des représentations sociales des élèves, des enseignants et des acteurs extrascolaires à propos de la lutte sénégalaise, implique aussi de mieux cerner les raisons et les motifs qui sont à l'origine du peu d'engouement de la lutte à l'école. Les raisons pouvant pousser les élèves à s'engager ou à apprendre la lutte scolaire. Ainsi nous définissons ci-après la lutte sénégalaise, objet de la représentation. Ensuite nous précisons les caractéristiques des représentations et leur utilité en didactique de l'éducation physique et sportive.

\section{1. La lutte sénégalaise}

3 Véritable patrimoine national, la lutte, appelée aussi Lamb ou Mbapatte, est pratiquée presque dans toutes les ethnies du Sénégal. Nous abordons l'activité à travers l'analyse de son environnement social et culturel premier : la société traditionnelle sénégalaise. L'ancrage culturel peut s'observer à travers les différents lieux et les moments de lutte, les modes d'entrée dans l'activité, les tenues vestimentaires, les pratiques magicoreligieuses, les techniques elles-mêmes. L'analyse de ces éléments et la mise en évidence d'une permanence autour des modalités de pratique nous renseignent sur la culture de l'activité qu'il conviendrait de transmettre à l'élève en EPS. Par lutte sénégalaise, nous entendons toutes les formes de lutte uniformisées et pratiquées au Sénégal, donnant lieu à des compétitions codifiées. Il s'agit notamment de la lutte avec frappe et de la lutte simple. Nous voulons par là nous différencier du terme "traditionnel», qui lui est toujours associée, qui nous semble péjoratif et de nature à limiter toute ambition de modernisation de la discipline.

\section{1. 1. La lutte traditionnelle simple, une pratique rurale}

4 La lutte sénégalaise moderne puise ses origines dans des luttes traditionnelles essentiellement rurales et communautaires. Pour mieux la comprendre nous nous proposons d'en voir les spécificités et le sens.

La lutte traditionnelle est l'expression d'une communauté ethnique, tribale ou clanique (Kalalobé, 1962, p. 34), qui honorent les croyances et les rites du terroir dans le respect des fonctions et divisions sociales. "Aux griots de battre les tambours, aux marabouts - «sorciers » de parler avec les « esprits » et les «djinns », aux femmes de chanter, ... et aux anciens "d'arbitrer» (Ly, 1996, p. 22). Les usages sociaux de la lutte traditionnelle simple sont festifs, rituels et même culturels, selon les ethnies. 
La réglementation varie en fonction des communautés. Si chez les Diolas, il faut terrasser son adversaire à deux reprises pour être déclaré vainqueur, chez les wolofs, une seule suffit. Dans le même sens, les prises, les gardes, les danses, etc., présentent des variantes selon la localité de référence. Selon Petrov «la technique, c'est l'armement du lutteur. Elle se subdivise en prises, parades, ripostes au moyen desquelles le lutteur va essayer d'accéder à la victoire. La technique détermine le style, la physionomie individuelle des lutteurs » (1984, p. 172).

La lutte simple est avant tout une activité visant par sa pratique à acquérir certaines valeurs allant dans le sens de la socialisation des individus. Elle s'impose presque partout comme un moyen de valorisation de l'honneur à travers le culte de la bravoure et confère à ses champions de village ou de contrée, un important capital social.

Mais la lutte simple ne rapporte que des trophées symboliques et une lutte plus lucrative s'impose progressivement autorisant dans le même temps la frappe aux poings. Les techniques traditionnelles sont donc réinterprétées au profit d'une lutte davantage codifiée que tous les sénégalais connaissent.

\section{1. 2. La lutte sénégalaise avec frappe, une pratique urbaine}

5 La lutte avec frappe semble donc être une synthèse de la boxe et de la lutte traditionnelle simple. Comme le stipule les règlements généraux de cette discipline « les lutteurs utilisent les techniques de la lutte sénégalaise, plus les coups de poing en usage dans la boxe anglaise » (article 28). C'est une activité qui occupe une place importante dans l'univers sportif de la capitale. La lutte avec frappe draine des foules, déchaîne des passions et est exploitée à des fins commerciales. Elle met en opposition les valeurs traditionnelles et modernes. Mieux médiatisée et pouvant permettre aux lutteurs de remporter d'importantes sommes d'argent, la lutte avec frappe est perçue aujourd'hui comme un moyen d'insertion socioprofessionnelle par beaucoup de jeunes. Elle se caractérise aujourd'hui par un perfectionnement rapide de la technique et de la tactique grâce surtout à l'affrontement de différentes écoles de lutte, appelées « écuries». La lutte avec frappe ne comporte pas de catégorisation de poids. Le combat se déroule généralement dans les stades de football, au milieu du terrain, sur le gazon ou sur une surface sableuse dans un espace de forme circulaire de 20 à 30 mètres de diamètre, délimité par des sacs remplis de sable. Il dure quarantecinq minutes, découpées en trois rounds de quinze minutes avec cinq minutes de repos entre chaque round. L'objectif fondamental de la lutte sénégalaise consiste à amener son adversaire au sol (assis, allongé au sol, ...). La victoire acquise a ainsi une valeur symbolique évidente. Le corps est la cible principale et le moyen de l'action. «Le combat est dominé par le principe tactique d'action - réaction-action » (FFL, 1981, p. 41). L'avant combat est marqué par un long ballet de rituels magico-religieux, de chants, de danses offrant à l'activité une dimension artistique, spectaculaire et culturelle qui intéresse les spectateurs au même titre que le face à face des deux lutteurs. Le lutteur et son entourage se voient dans l'obligation de se soumettre à ces rituels à des fins de protection, mais aussi pour se rassurer. En ce sens, le marabout occupe une place importante. Il joue le rôle de préparateur mental. Ses pouvoirs et son influence sont parfois même plus forts que ceux de l'entraîneur.

Très populaire, la lutte avec frappe, se présente de plus en plus comme un mode de socialisation dans les villes, qui offre une profession à des centaines de jeunes ruraux qui viennent chercher une hypothétique insertion sociale. Le déplacement des 
pratiques de lutte vers les centres urbains, pénétrés par la professionnalisation et l'occidentalisation est déterminant dans la transformation du sens de ces pratiques. Ces pratiques qui étaient anciennement comprises dans les jeux traditionnels développent aujourd'hui une logique de sportivisation au regard de leur théâtralisation en terme d'opposition, de compétition, de spectacle, de production d'images et de leur institutionnalisation à travers des structures fédérales.

L'analyse souligne donc que la lutte, malgré son évolution et sa sportivisation, est bien une pratique ancrée dans sa culture. La lutte sénégalaise s'explique et se comprend dans un contexte local où valeur, tradition, religion et économie contribuent à l'unité communautaire. Cette analyse nous a permis d'identifier plusieurs variables fondamentales que nous répartissons en trois catégories:

- Les croyances et les rites. Les luttes traditionnelles possèdent au départ une fonction de fête ou de loisir souvent en relation avec des manifestations religieuses. Les pratiques rituelles déterminent l'organisation de la vie autour des croyances (compréhension du sens des pratiques mystiques, magico-religieuses, etc.), les modes d'entrée dans l'activité, les manières de danser, de s'habiller, de faire ;

- Les enjeux économiques et financiers. La codification des règles et l'accroissement des cachets ont entrainé la sportivisation de la lutte et le professionnalisme ;

- Les techniques, qui caractérisent des principes d'exécution, des principes technico-tactiques et stratégiques.

Ces différentes catégories vont donc constituer notre grille d'analyse pour comprendre la lutte sénégalaise, de sa forme traditionnelle jusqu'à sa forme sportive (professionnelle). En effet, les enjeux de la lutte apparaissent étroitement liés, voire interdépendants. Le politique ne peut être dissocié de l'économique sur lequel il axe ses stratégies. Le social se partage entre le culturel et l'économique. Le culturel semble devenir un faire-valoir pour l'économique. Étudier la lutte sénégalaise, c'est étudier les sénégalais et leurs comportements face à leur culture, leur patrimoine, leur histoire, leur identité locale.

\section{1. 3. La lutte en EPS au Sénégal}

$7 \mathrm{Au}$ regard du développement de la lutte sénégalaise et de l'engouement qu'elle suscite chez les jeunes, nous nous interrogeons à propos des stratégies et contenus de formation pour le système éducatif (école), voire pour les « écuries» (club) de lutte. C'est donc le problème de l'identité culturelle, comme source d'inspiration de l'action didactique, qui est revendiquée ici. Il s'agit de lier l'action pédagogique au contexte culturel afin de la rendre plus efficace, plus attrayante. Nous pensons que l'éducation physique et sportive peut contribuer à une renaissance centrée sur les cultures ancestrales et les valeurs de civilisation en apportant aux contenus un enrichissement et un cachet d'affirmation culturelle. Cependant, si l'on se réfère aux instructions officielles (recueil des textes réglementaires relatifs à l'EPS) aucune indication, aucune référence n'est faite aux jeux traditionnels et à la lutte. Même dans les annexes où l'on parle des sports de combat, la lutte n'est pas évoquée. Ces dernières, sous l'influence de la loi d'Orientation de l'Éducation Nationale (dans sa version n ${ }^{\circ}$ 71-36 PM.SGG.SI du 3 juin 1971), précisent en son article 3 que « l'Éducation Nationale Sénégalaise est une éducation africaine, prenant sa source dans les réalités africaines et aspirant à l'épanouissement des valeurs culturelles africaines... ».

S'il existe des orientations dans le sens de l'intégration des jeux traditionnels et des 
pratiques corporelles, elles ne sont pas prises en compte dans les Instructions Officielles qui datent de 1973. Ce sont les États Généraux de 1981 et les travaux du CNEPS (Centre Nationale d'Éducation Populaire et du Sport) de Thiès (recueil des jeux traditionnels), qui formulent ces recommandations.

Ainsi, "la lutte, discipline sportive éminemment éducative, a été ignorée par le législateur scolaire, sans doute par suite de son appartenance aux activités traditionnelles et ses liens avec certaines catégories socioéconomiques peu représentées à l'école »(Programme d'implantation de la lutte à l'école comme discipline éducative, 1983). Depuis 1983, la lutte simple est inscrite dans les programmes comme discipline éducative. Mais jusqu'à présent, elle peine à avoir toute sa place à l'école. Dans les établissements, il est très rare de voir la lutte programmée dans les cycles d'enseignement de l'EPS. Elle ne figure ni dans les épreuves du Brevet de Fin d'Études Moyen (BFEM), ni dans les épreuves du Bac. La lutte est « délaissée » au profit des sports comme le football, le basket, le volley, la gymnastique. Pourtant, son enseignement, contrairement aux autres disciplines scolaires, nécessite peu de moyens (financiers et infrastructurels). En effet, la lutte peut être enseignée soit dans une salle de gymnastique ou de sports de combat (judo par exemple), soit sur une surface sableuse très accessible, car la plupart des établissements scolaires dispose de terrains vagues. Le climat aussi est favorable $\left(15^{\circ} \mathrm{C}\right.$ au minimum en hiver). La lutte renferme plusieurs éléments constitutifs tels que le symbolique, le rythme, le mouvement et l'expression du corps (forme), l'espace, le temps, qui font qu'elle constitue un instrument d'éducation des jeunes.

\section{1. 4. Problématique}

8 Fort de cette approche plurielle de la culture sénégalaise de la lutte, nous nous interrogeons sur les éléments de cette culture « enseignables » à l'école. Quelle forme de lutte scolaire serait-il possible d'enseigner à l'école au regard des finalités de l'Institution et des connaissances scientifiques et sociales disponibles? Cela nécessite d'une part, « une découverte de la structuration des savoirs et des savoir-faire présents, utilisables dans l'activité lutte pour les dépersonnaliser et les organiser dans une logique opératoire pouvant se matérialiser dans une représentation transmissible. Et d'autre part, cela suggère une prise en compte des niveaux de pratique, le repérage des logiques de comportements et d'appropriation des savoirs et des savoir-faire par combattant (lutteur) et les transferts possibles » (David, 1996, p. 12).

Pour Marsenach et Mérand (1987, p. 25), «les contenus d'enseignement sont les conditions à intérioriser, qui permettent l'élaboration d'actions nouvelles, elles-mêmes corrélatives de transformation de l'activité corporelle». Ce sont «les conditions que l'élève doit intégrer pour transformer ses actions (Marsenach, 1991, p. 44). Remarquons que les «conditions évoquées» ici renvoient bien à l'idée d'une organisation externe du sujet apprenant, qui peut intérioriser des savoirs par le biais de situations et contenus d'apprentissage proposés par l'enseignant. Ce dernier accumule des savoirs pratiques, fruits de l'expérience professionnelle, en construisant des théories d'action personnelles dérivées, des théories d'expert et parfois de données scientifiques. Il convertit alors ces données en cycle, exercices, situations, constituant ainsi un répertoire de contenus à enseigner en fonction de la population scolaire qu'il a en responsabilité et des conditions matérielles et géographiques.

Pour mieux comprendre l'activité de l'élève confronté à l'enseignement de la lutte, un 
axe possible consiste à approcher les représentations sociales de la lutte sénégalaise des élèves, celles des enseignants et celles des acteurs extrascolaires. L'idée est de faire ressortir les points saillants des représentations sociales d'une activité physique pratiquée régulièrement par la majeure partie de la population sénégalaise, d'en étudier la nature et d'en estimer l'importance, afin d'identifier :

- en quoi ces représentations peuvent constituer un frein ou un élément d'explication quant à la « quasi » non pratique ou le peu d'engouement de la lutte en EPS ;

- en quoi ces représentations sociales peuvent nous aider à donner du sens à l'activité et faire percevoir ses enjeux de formation ;

- en quoi ces représentations sociales peuvent nous orienter dans notre élaboration de contenus en lutte scolaire.

9 Avant de présenter la méthodologie de notre travail et les données empiriques relatives aux représentations sociales et fonctionnelles des acteurs scolaires et extrascolaires de la lutte sénégalaise, nous croyons nécessaire d'en reprendre les caractéristiques essentielles et d'en mesurer l'impact pour les apprentissages.

\section{2. Caractéristiques des représentations sociales}

10 Hétérogène et polysémique, le concept de représentation sociale tire son origine au sein de la sociologie durkheimienne plus particulièrement autour de la notion de représentation collective. Les représentations sociales ont depuis nourri tout un courant de recherche porté notamment par Moscovici, Herzlich, Kaes, Jodelet et Abric. Les recherches sont d'autant plus complexes qu'elles se situent au carrefour de plusieurs disciplines, soit la sociologie, la psychologie sociale, l'anthropologie et l'histoire. «Les représentations sociales sont des modalités de pensée pratiques orientées vers la communication, la compréhension et la maitrise de l'environnement social, matériel et idéel»(Jodelet, 1989, p. 361). Elles sont généralement marquées affectivement, ce qui contribue à expliquer le poids dont elles peuvent peser sur les attitudes et l'engagement des sujets dans les activités. La représentation apparaît donc comme " un ensemble organisé d'opinions, d'attitudes, de croyances, et d'informations se référant à un objet ou une situation. Elle est déterminée à la fois par le sujet lui-même (son histoire, son vécu), par le système social et idéologique dans lequel il est inséré et par la nature des liens que le sujet entretient avec ce système social» (Abric, 1999, p. 206). Mannoni (1998) nous rappelle qu'elle se joue sur une triple scène :

- la scène 1 , constituée par l'imaginaire individuel où apparaissent les représentations individuelles (images, vécus, fantasmes) ;

- la scène 2, qui est celle de l'imaginaire collectif et où apparaissent véritablement les représentations sociales (du cliché et du préjugé aux contes et aux mythes);

- enfin la scène 3 , composée de la réalité sociale agie, où se manifeste les actions socialement représentées.

11 Le concept de représentation sociale est si riche et si complexe qu'il n'est pas toujours évident de le définir. Pour arriver à cerner cette notion, il est nécessaire d'ordonner et de schématiser son contenu. En tant que savoir pratique de sens commun, les représentations sociales ont plusieurs fonctions : catégorisation cognitive des objets, d'identification sociale et individuelle, d'orientation et de prescription des comportements, de référentiels ou de gisements de savoirs pour les justifications ou les 
rationalisations (Seca, 2002).

L'étude des représentations sociales s'est enrichie depuis ces dix dernières années de théories, de modèles et de techniques de mesure qui permettent aujourd'hui, aux différents chercheurs concernés, la prédiction et la validation expérimentale.

Abric $(1987,1994)$ et Flament $(1989,1994)$, ont développé un modèle théorique qui caractérise la représentation sociale comme étant formée d'un système central (noyau structurant de la représentation) et d'un système périphérique. Selon Abric (1994), les représentations sociales possèdent une double caractéristique : elles sont à la fois stables et mouvantes, rigides et souples. Elles sont stables et rigides, parce que déterminées par un système central profondément ancré dans le système de valeurs et de normes partagées par les membres du groupe. Les éléments qui constituent ce système central définissent des principes fondamentaux autour desquels se constituent les représentations et jouent donc un rôle essentiel dans la stabilité et la cohérence de celles-ci. Ils sont de plus, indépendants du contexte immédiat dans lequel le sujet utilise ou verbalise ses représentations. Mais les représentations sont aussi mouvantes et souples, car nanties d'un système périphérique composé d'éléments dont la détermination est plus individualisée, et surtout associée au contexte contingent dans lequel baignent les individus. Le système périphérique permet ainsi une adaptation, une différenciation en fonction du vécu et une intégration des expériences sociales quotidiennes. Les éléments centraux se présentent donc comme insensibles aux variations de contexte, à l'inverse des éléments périphériques (Guimelli, 1996).

Les représentations sociales constituent ainsi, une grille de lecture à travers laquelle on décode une certaine dualité. Cette grille, qui est constituée de tout un héritage sociohistorique, nous est léguée dès notre naissance à travers les multiples interactions et communications que l'on entretient avec notre entourage proche et éloigné. En même temps, il appartient à chacun de se réapproprier cet héritage, de le construire pour qu'il acquière un sens personnel. Ainsi, «les représentations sociales sont des principes générateurs de prises de position liées à des insertions spécifiques dans un ensemble de rapports sociaux et organisant les processus symboliques intervenant dans ces rapports » (Doise, 1986, p. 89). Autrement dit, ce n'est pas tant la réalité objective de l'objet (l'activité physique par exemple) ou de la situation qui oriente les propos et détermine les conduites des individus, mais la représentation qu'ils en font. Ici, ce n'est pas la signification de l'activité qui compte mais plutôt les représentations induites par celle-ci. Comme le souligne Rouquette (1994, p. 174), « une représentation sociale est susceptible d'avoir elle-même des effets sociaux, repérables dans les pratiques et les conduites de la population (...). Ce n'est pas que l'on agisse conformément à ce que l'on pense, mais ce que l'on pense balise l'action possible ». La pratique ou la non pratique d'une activité physique peut être donc liée aux représentations sociales que l'on s'en fait. Cela nous amène par conséquent à analyser sa place en didactique des APSA.

\section{3. Les représentations en didactique des APSA et didactique de I'EPS}

Différents auteurs ont cherché successivement à identifier, comprendre, expliquer et utiliser le concept de représentation dans l'enseignement des disciplines scientifiques (Giordan et De Vecchi, 1987; Astolfi et Develay, 1989; Astolfi, 1992). Comme le soulignent Cornu et Vergnioux (1992, p. 50), aujourd'hui dans toutes les didactiques, « les chercheurs ont mis en évidence que le point de départ obligé de tout 
apprentissage devait être la prise en considération et le recueil, l'inventaire des représentations ou conceptions présentes dans l'esprit des élèves, leur système de savoir ». Ainsi, «ce concept (représentation) nomade capté par la didactique » selon Develay (1992, p. 78), «favorise une approche nouvelle de l'apprentissage susceptible d'expliquer la manière dont nous construisons le réel». En didactique de l'EPS, une approche rationnelle est exigée de la part des enseignants, afin qu'ils effectuent non seulement le choix des APS, mais également le choix des objets d'enseignement puis, des contenus d'enseignement ainsi que des modalités de leur mise en œuvre. Cependant, comme le soulignent Irlinger, Louveau et Metoudi (1992, p. 54) les APS «bénéficient par ailleurs d'autres modes de transmission plus informels, via la télévision, la famille, l'environnement en général ». Les enseignants d'EPS sont alors confrontés à l'hétérogénéité des connaissances des élèves. Cela aura pour conséquence un accroissement des travaux portant sur l'articulation entre représentations et apprentissages en EPS.

Ces travaux ont permis de "dresser un état descriptif des représentations et des productions motrices chez les élèves d'âge, de sexe, de niveaux d'expertise différents dans les APS scolaires" (Marsenach, Amade-Escot, 1993, p. 36). D'autres recherches essaient d'analyser les effets des représentations de leur propre compétence chez les élèves, sur leur motivation et leur engagement dans les tâches sportives en EPS. Pour l'essentiel ces travaux semblent considérer que le niveau d'habileté motrice des élèves et leurs capacités à apprendre en EPS dépendent entièrement des processus cognitifs impliqués dans les apprentissages.

Les recherches de Bouthier (1993), David (1993, 1995, 1996), Aubert (1997) sur les représentations sociales des élèves en EPS, ont mis en évidence les influences de la pratique sur les représentations initiales d'élèves concernant des APS variées. Ces études permettent d'apprécier les cohérences entre, conception et connotations de l'activité, buts et actions valorisés au plan déclaratif, et engagement et conduites d'élèves dans la pratique.

L'analyse des représentations, dans la mesure où elle pourrait être réalisée aisément par l'intervenant, comme le précise Bouthier (1993), est une source intéressante d'informations pour planifier et réguler l'enseignement ou l'entraînement, notamment dans la perspective de mise en œuvre de projets et de contrats (dimension qui nous intéresse). Elle peut influencer les contenus d'enseignement et d'entraînement, les progressions proposées, en tenant compte des représentations fonctionnelles des sujets, révélatrices de leurs logiques d'action et des obstacles qu'ils rencontrent. Elle peut intervenir, à partir de l'évolution constatée des représentations, pour refléter le niveau d'appropriation et de reconstruction de certaines connaissances au regard des réalisations motrices produites. Cette analyse peut nous permettre de comprendre la signification des comportements adoptés par les élèves et les enseignants face à la lutte sénégalaise. L'approche des représentations sociales présente pour nous un intérêt certain dans la mesure où le regard porté par les élèves, les enseignants d'EPS, voire les parents sur les différentes formes de luttes au Sénégal, peut nous permettre de déterminer la relation entre leurs représentations sociales des luttes et des pratiques de lutte existantes ou possibles. Ainsi, considérant les constats théoriques au niveau des représentations sociales notamment en didactique de l'EPS, nous allons à présent identifier la nature des représentations sociales des élèves, des enseignants et des acteurs extrascolaires de la lutte sénégalaise. 


\section{Méthodologie}

13 représentations.

L'étude des représentations sociales nécessite l'utilisation de méthodes spécifiques dans le but d'identifier le contenu et la structure de la représentation. Selon Aubert (1997, p. 155), « les travaux existants indiquent que l'inférence à partir de l'expression verbale ou figurative des individus est le moyen le plus utilisé ». Dans le cadre de notre étude, nous mobilisons deux techniques pour recueillir le discours des différents acteurs : un questionnaire pour les enseignants et les élèves et un entretien semi directif pour les acteurs extrascolaires. Les objectifs sont de mieux connaître les opinions, les croyances, les comportements et les connaissances des différents acteurs par rapport à la lutte. Le questionnaire, tout comme le guide d'entretien, est composé de plusieurs parties articulées selon une logique qui débute par l'identité du sujet pour aller vers leur intérêt pour la lutte, en passant par leur connaissance et le sens attribué à la discipline. Concernant les parties relatives aux significations et intérêts pour la lutte, nous avons préféré proposer des questions ouvertes plus à même d'approcher le point de vue des protagonistes.

Pour les acteurs scolaires, nous travaillons sur 192 questionnaires élèves et 35 enseignants des collèges et lycées de la capitale et de l'intérieur du pays. Le taux de retour est de plus de $70 \%$. Les 23 acteurs extra scolaires se composent de 6 lutteurs, 5 membres du Comité National de Gestion de la Lutte (CNGL), 3 arbitres, 2 griots, 2 historiens, 2 sages ( 1 marabout et 1 ancien lutteur), 1 chanteuse, 1 promoteur et 1 manager. Le recours à des entretiens semi directifs plutôt qu'à des questionnaires tient aussi au type de public auquel nous nous adressons. En effet le monde de la lutte est très fermé et les croyances magico religieuses rendent très susceptibles les acteurs. Nous avons par ailleurs, pour certains, utilisé le wolof, langue nationale, pour conduire les interviews. Il nous apparaît que la connaissance fine du milieu et l'immersion du chercheur dans le contexte des acteurs permet de recueillir des données originales difficiles à obtenir avec d'autres méthodes.

Les réponses apportées aux questionnaires et aux entretiens nécessitent une analyse de contenu dont le but est l'inférence de connaissances relatives aux conditions de production ou éventuellement de réception, à l'aide d'indicateurs quantitatifs ou non (Bardin, 1993). L'inférence est la procédure intermédiaire qui permet le passage explicite et contrôlé de la description (résumée après traitement) à l'interprétation (phase ultime : la signification accordée aux caractéristiques).

Pour les questions ouvertes l'analyse de contenu est effectuée en utilisant le logiciel Sphinx, qui consiste à lire chaque réponse pour rechercher les thèmes qu'elle contient et la décrire en cochant les modalités d'une nouvelle variable fermée, sur la liste des thèmes rencontrés à partir de la séquence "recoder : coder des textes » (Sphinx, 1995, p. 230). Cette solution, bien que longue, nous semble intéressante pour recueillir le sens du contenu des textes et nous offre plus de possibilités. Nous ne pouvons nous contenter, ici, d'une analyse lexicale et d'extraits de verbatim trop réducteurs et peu adaptés à notre étude.

S'agissant des entretiens semi-directifs nous avons opté pour l'analyse thématique (Blanchet, 1991 et Muchelli, 1996), qui repose sur une interprétation des énoncés. Elle cherche une cohérence thématique inter entretiens. Les thèmes sont, ici, 
des unités de signification, des noyaux de sens. Ces outils permettent d'approcher l'étayage des représentations sociales des acteurs scolaires et extrascolaires. Nous présentons maintenant les principaux résultats. Ensuite, nous comparons ces représentations afin de dégager les éléments pouvant nous aider à l'élaboration de contenus en lutte scolaire et dans le choix des modes d'entrées dans l'activité.

\section{Résultats}

Dans cette partie, nous analysons les caractéristiques les plus marquantes des représentations sociales de la lutte sénégalaise. Nous les considérons comme des éléments centraux des représentations des différents acteurs. Les résultats sont présentés en trois temps : connaissance de l'activité, signification de la lutte et l'intérêt des différents acteurs pour la lutte. Concernant la signification et l'intérêt pour la lutte, nous les organisons en 3 catégories :

- des aspects culturels, rituels de l'activité ;

- des aspects économiques relatifs à la sportivisation de l'activité ;

- des aspects technico-tactiques et stratégiques de la lutte, etc.

\section{1. Signification de la lutte}

$96,7 \%$ des élèves et $100 \%$ des enseignants connaissent la lutte. Les élèves qui déclarent ne pas connaître la lutte sénégalaise sont en majorité d'origine étrangère vivant à Dakar.

Tableau I. Signification de la lutte

\begin{tabular}{|l|l|l|}
\hline Appréciations & Enseignants & Élèves \\
\hline Positives & $50,8 \%$ & 78,9 \\
\hline Négatives & $39,7 \%$ & $17,6 \%$ \\
\hline Autres & $9,5 \%$ & $3,5 \%$ \\
\hline Total observé & $100 \%$ & $100 \%$ \\
\hline
\end{tabular}

Les résultats exprimés dans le tableau I indique les différents choix de réponses à la question "que pensez-vous de la lutte». Il est à remarquer que les pourcentages les plus élevés aussi bien pour les enseignants que pour les élèves concernent les aspects positifs de la lutte. Les motifs invoqués sont notamment des enjeux économiques (devenir riche, avoir un métier, avoir de l'argent, développer le pays), des significations culturelles (valoriser la tradition, la culture, le patrimoine, développer un sentiment de fierté, défendre un sport national, être original) et de l'évolution de la lutte au Sénégal (très développée, très aimée, très intéressante, très respectée). Les appréciations négatives renvoient à une perception de la lutte comme étant une activité dangereuse, violente et qui dénature la lutte simple sans frappe. S'agissant des appréciations " autres ", chez les enseignants comme chez les élèves, elles s'articulent autour des éléments suivants : «ils souhaitent une suppression de la frappe qui pour 
eux, est dangereuse et ne permet pas de voir la technique pure du lutteur. Les acteurs scolaires admirent le courage et la morphologie des lutteurs. Ils estiment aussi que la différence de poids des lutteurs nécessite une réglementation ».

Pour les acteurs extrascolaires, les réponses fournies lors des entretiens montrent que la lutte est considérée comme une activité noble, de "chez nous", un héritage à transmettre. Elle fait partie de l'éducation du jeune sénégalais. En témoigne ce point de vue du président du Comité National de Gestion de la Lutte (CNGL) « Ce que je pense de la lutte, c'est ce que pensent les Sénégalais en général. Cela veut dire que c'est un sport bien de chez nous, surtout la lutte avec frappe qu'on ne retrouve nulle part ailleurs à travers le monde (...) qui, depuis quelques années, prend de l'ampleur, commence même à se professionnaliser. Ainsi il permet à une partie de notre jeunesse, qui malheureusement à cause des échecs scolaires ou de l'absence de scolarisation, pourrait devenir des agresseurs ou autres, d'exprimer de façon positive cette violence qui est dans tout individu et à partir de là, gagner honnêtement leur vie, et surtout se valoriser par rapport à cette société à problème » (Extrait entretien avec le Président du CNGL). Ce témoignage corrobore les propos du responsable de la lutte traditionnelle sans frappe « la lutte, c'est ce que nous avons au Sénégal. C'est le seul sport national non importé. Et je pense qu'on a bien intérêt à le développer et à le divulguer, que ça sorte de nos frontières (...). Jusqu'à présent, je dirais: un lutteur c'est un notable. Si je reprends un peu l'histoire de la lutte, c'était des guerriers au départ, des gens qui faisaient la guerre. C'est pour cela que c'est entre villages. Un problème d'honneur. Donc, ce n'est pas comme ce que certains pensent du lutteur : un crétin, un idiot. Non, ils étaient parmi les gens les plus intelligents, les plus intégrés et les plus costauds. Donc la lutte a une grande signification pour moi et je suis sûr que dans quelques années, je le dis et les gens ne me croient pas beaucoup, les grands lutteurs seront des universitaires comme aux USA où les grands basketteurs sont des universitaires. Pour vous dire que ce ne sont pas des idiots » (entretien avec le responsable de la lutte sans frappe).

Nous relevons également des réponses plus spécifiques du statut des acteurs extrascolaires.

\section{1. 1. Représentations des lutteurs, des promoteurs et des managers}

17 Pour les lutteurs ce sport est un moyen d'insertion sociale. Ils insistent fortement sur les possibilités financières qu'offre la lutte avec frappe. Selon le lutteur JB, « la lutte faisait partie de notre éducation. Mes parents aussi étaient lutteurs. Ils leur arrivaient d'organiser des combats pour les enfants avec comme récompense des bonbons, des biscuits, etc. La lutte était notre jeu favori... j'aime la lutte. Ensuite, je crois que c'est le moyen pour moi de gagner ma vie et d'aider mes parents" (entretien avec Junior, lutteur d'ethnie sérère). N'Diaye, quant à lui précise que «la lutte joue un rôle important dans notre pays. Elle a participé fortement à la diminution des agresseurs. Parce que beaucoup d'entre eux sont devenus lutteurs... L'image du lutteur a beaucoup évolué. Parce qu'avant, les lutteurs étaient classés et non considérés. Mais aujourd'hui, presque tous les parents souhaitent voir leur fils devenir lutteur. Pourquoi ? À cause du succès, de l'argent qu'on peut gagner dans la lutte» (entretien avec N'diaye lutteur d'ethnie toucouleur, de l'écurie Pikine Mbolo ).

Cette conception est partagée par les promoteurs et les managers pour qui la lutte est devenue un business. "Le promoteur de lutte doit (mettre en avant) plus favoriser 
l'épanouissement et le développement du pays que ses intérêts financiers. Je pense que le fait de rassembler des jeunes qui, peut-être, allaient devenir des délinquants, les récupérer et les amener dans la lutte afin qu'ils puissent s'en sortir et gagner leur vie, c'est important. Donc, le promoteur doit être un patriote, qui aime son pays... À chaque fois que vous voyez les combats de 75 millions CFA, cela veut dire qu'il y a un grand sponsor derrière qui vient, qui pose de l'argent sur la table pour que le promoteur puisse organiser le combat. Parce que l'organisation de combats nécessite de l'argent»(entretien avec un promoteur de lutte sénégalaise). De son côté le manager que nous avons rencontré pense qu'« ...aujourd'hui les lutteurs sont malins. Avant le lutteur était seul, quand il parlait on ne le considérait même pas, mais actuellement les lutteurs sont devenus éveillés. Chacun a sa personne de confiance qui te guide, te coache, te conseille de telle sorte que maintenant les lutteurs comprennent presque tout. Aujourd'hui, les lutteurs se baladent en Pajero, ils mettent des cravates alors que cela n'existait pas avant. Le principal rôle des promoteurs est d'organiser des combats. Ils viennent au stade, ils regardent les combats, les meilleures affiches et les programment. Ils sont là pour développer certes la lutte, mais aussi pour se faire de l'argent. Parce que quand on parle de promoteur, c'est pour gagner de l'argent mais aussi il ne faut pas oublier que le plus souvent les promoteurs sont des amateurs. Ils participent aussi au développement de la lutte et en même temps, ils gagnent de l'argent. L'argent est surtout leur première source de motivation ». Ici l'aspect économique est mis en avant.

\section{1. 2. Représentations des responsables administratifs du CNGL, des historiens et animateurs}

18 Pour les membres du CNGL (président, secrétaire général, responsable lutte amateur et médecin), les historiens et les griots, la lutte est le sport national. Ils insistent sur les nombreuses mutations de la discipline. Les membres du CNGL mettent en exergue la gestion qu'ils ont mises en place et qui a amené une certaine stabilité. Les historiens et les griots insistent sur l'évolution des pratiques rituelles, les modes d'entrée dans l'arène, les tenues vestimentaires et la place de plus en plus importante de la frappe au détriment de la technique de lutte pure. Selon Ly (historien) « l'évolution de la lutte peut être étudiée ou envisagée sous des angles extrêmement divers. Prenez l'habillement des lutteurs, il y a une cinquantaine d'années quand le lutteur venait, il avait son accoutrement. Il avait ses pagnes, ses ngebm (pagne enroulé autour de la taille que le lutteur fait ensuite passer entre ses jambes pour faire un nœud), il avait tout. Aujourd'hui, tel n'est plus le cas. Un grand lutteur comme Tyson, quand il a un grand combat, il arbore son drapeau. C'est dommage, parce qu'avant, on pouvait identifier chaque lutteur de par le rythme du tam-tam et on savait quel genre de lutteur venait : est-ce un Sérère? Est-ce un Wolof ou un Diola? Ou est-ce un Peuhl ? On le savait avant même qu'il n'arrive par les sons des tam-tams. Et quand il arrivait, si vous ne connaissiez pas son nom, par son accoutrement, vous connaissiez l'ethnie à laquelle il appartenait. Mais de plus en plus, tout a tendance à vraiment se niveler vers la monotonie, ça devient purement du commerce. Mais encore quel commerce? Le lutteur d'aujourd'hui, c'est quelqu'un qui cherche de l'argent. C'est un professionnel. N'importe quel garçon qui se développe, a des muscles, va aller dans une écurie et se dit je vais chercher des millions. C'est son droit le plus absolu. Mais avant même l'indépendance, c'était traditionnel et chaque terroir, chaque chef de canton ou 
chaque chef régional avait son lutteur qui représentait toute la contrée comme l'équipe nationale. La technique est maintenant reléguée au second plan avec la frappe. Il y avait tellement de champions de lutte avec des techniques extraordinaires. Vous qui êtes professeur, je pense qu'il faut que vous cherchiez dans le vocabulaire d'antan, parce qu'il y a beaucoup de choses qu'on n'entend plus. Des techniques qu'utilisaient les grands lutteurs, par exemple : Nodio. Ça fait longtemps que je n'ai pas $\mathrm{vu}$ un lutteur utiliser cette technique dans l'arène » (entretien avec Ly historien).

\section{1. 3. Représentation des cadres techniques du CNGL}

19 Le Directeur Technique National (DTN) et l'entraîneur national précisent que les jeunes lutteurs ne font pas assez la lutte simple. Ils veulent embrasser très tôt la lutte professionnelle. Ce choix s'explique pour eux par le fait que la lutte traditionnelle n'est pas très bien valorisée aujourd'hui contrairement à la lutte avec frappe où les cachets sont devenus très lucratifs. «La lutte est aujourd'hui championne d'Afrique je ne sais combien de fois. Il n'y a qu'une seule fois, si mes souvenirs sont exacts, où un Ministre de la République a décoré les lutteurs. Moi personnellement, c'était la seule fois où j'ai été décoré. Aujourd'hui, il y a un texte au niveau du Ministère qui est sorti qui dit que si vous avez une médaille en or, vous avez tant, médaille d'argent, tant, etc. Mais il n'y a rien pour l'encadreur. Il y a problème car quand on est en regroupement, c'est l'entraîneur qui prend de sa poche pour subvenir aux besoins de ses lutteurs » (entretien avec le Directeur Technique National de la Lutte). Concernant les arbitres, ces derniers déplorent la méconnaissance du règlement par les lutteurs, leur staff et le public. L'un d'entre eux témoigne: «le problème majeur, c'est la réglementation. Et puis, les lutteurs sont indisciplinés, le cercle de lutte est indiscipliné, il y a un règlement qui est bel et bien établi mais qu'ils ne respectent pas. Je ne sais pas s'ils le connaissent, s'ils font la tête mais ils ne l'appliquent pas. C'est très difficile " (entretien avec un Arbitre de lutte). "C'est vrai, il y a des lutteurs qui ne connaissent pas le règlement, précise N'diaye le lutteur ! Même moi, il y a des choses que je connais dans le règlement parce qu'en prenant la licence de lutteur, je dois être en mesure de connaître mes droits et mes devoirs. Mais sincèrement, je ne connais pas tout le règlement. Il y a des choses que je maîtrise et d'autres que je ne connais pas ».

L'analyse des significations de la lutte sénégalaise met en exergue les liens qui s'établissent entre les pratiques rituelles du sport et les dimensions économique, politique et sociale. La lutte génère au niveau des différents acteurs des moyens d'affirmation identitaire notables. La mise en spectacle des manifestations de lutte produit des signes émouvants et des images identifiantes qui sont autant d'éléments pouvant susciter l'engouement d'une communauté et favorisent la conscientisation des décideurs, hommes politiques, pratiquants, réseaux associatifs. La lutte valorise les traditions et apparaît comme un véritable moyen d'insertion sociale. Cependant, l'organisation des combats par des promoteurs, des managers du sport, gérant les événements comme une entreprise commerciale à la recherche de profits, génère certes une manne économique assez importante, mais risque de déstructurer les symboles initiaux. Les significations originelles de la lutte peuvent être perturbées par l'emprise économique. 


\section{2. Intérêts pour la lutte}

Concernant leurs préférences, $58 \%$ des enseignants et $62 \%$ des élèves encouragent la lutte simple sans frappe contre respectivement $21 \%$ et $28,44 \%$ pour la lutte avec frappe. Mais il s'agit de savoir ce qu'ils préfèrent vraiment dans l'activité lutte.

Tableau II. Intérêt pour la lutte

\begin{tabular}{|l|l|l|l|}
\hline Intérêts & Enseignants & Élèves & Acteurs extrascolaires \\
\hline Aspects culturels et économiques & $40,4 \%$ & $50,2 \%$ & $70 \%$ \\
\hline Aspects technico-tactiques et stratégiques & $34 \%$ & $41,4 \%$ & $30 \%$ \\
\hline Autres & $25 \%$ & $8,4 \%$ & $0 \%$ \\
\hline TOTAL & $99,4 \%$ & $100 \%$ & $100 \%$ \\
\hline
\end{tabular}

Le tableau II représente une compilation des réponses des enseignants et des élèves à la question « qu'est-ce qui vous plait dans la lutte?». Les aspects culturels (ambiance, chants, habillement et danses des lutteurs, pratiques mystiques, signatures de combats) et économiques (argent, montant des cachets) représentent les éléments les plus appréciés des enseignants $(40,4 \%)$ et des élèves (50,2 \%). Leur intérêt se portent ensuite sur les aspects techniques, tactiques et stratégiques de l'activité pour $34 \%$ des enseignants et $41,4 \%$ des élèves (corps à corps, manières de terrasser, la frappe, engagement des lutteurs).

Pour les acteurs extrascolaires, le spectacle prime comme le précise le promoteur de combats de lutte: «quand on parle vraiment de lutte sénégalaise, c'est la culture, il est sûr que j'accorde une grande place à ces pratiques. Déjà mon souhait, c'est, avec l'aide de Dieu, partout où j'organise, que les gens disent qu'ils n'ont jamais assisté à une manifestation aussi intéressante. De ce fait, je réunis toutes les conditions, c'est à dire : pratiques culturelles, tradition et sport. J'essaie d'amener des danseurs traditionnels, j'essaie d'intégrer toutes les pratiques culturelles qui gravitent autour de cette zone, localité pour que tout se passe bien » (entretien un promoteur). «La lutte ne peut pas exister ou se développer sans back, sans les aspects rituels (danse, chant, pratiques mystiques, etc.). Pour les anciens lutteurs, ce qui faisait leur force et leur notoriété, c'est parce qu'ils savaient faire des backs » (entretien avec un Griot, Animateur et commentateur de lutte à la Radio Télévision Sénégalaise). «Les backs, des chants, des danses, c'est l'ambiance de la lutte. La danse permet de voir la notoriété du lutteur. La lutte vient de Dieu, et c'est une activité noble. Les chants permettent de stimuler le lutteur. Avant, chaque lutteur avait ses chanteurs, ses propres chansons qui lui rappelaient son origine, l'histoire de ses ancêtres, sa localité, la bravoure de ses parents, de ce qu'on attend d'un lutteur, la symbolique de la lutte » (entretien avec Gorgui, ancien lutteur né en 1924, actuellement marabout de profession). 


\section{3. Les pratiques magico religieuses}

Dans les rencontres de football, le règlement interdit les pratiques occultes. Et toute l'équipe qui est prise en train de verser des poudres, d'enterrer des gris-gris, etc., est immédiatement sanctionnée. Par contre, au niveau du lutteur, c'est l'absence de ces pratiques qui est sanctionnée. Le public va juger de l'intensité, du sérieux du combat en rapport avec la préparation mystique. Ainsi, on parle de combats arrangés à l'avance pour tout lutteur qui se présente à l'arène de manière très rapide pour faire sa préparation mystique. Ce qui amène Kane enseignant d'histoire de l'EPS à l'INSEPS de Dakar à dire que « c'est un élément constitutif de cette pratique. Et dans le langage, les lutteurs, quand on les interroge, disent que je suis physiquement prêt, tactiquement prêt, techniquement prêt et mystiquement prêt, dire que c'est une fonction fondamentale. Et d'ailleurs, un lutteur comme MF, vous lui demandez quelle est la part du marabout dans un combat maintenant que vous avez arrêté de lutter. Il dit que qu'autant il faut s'entraîner mais autant la préparation mystique est fondamentale » (entretien avec Kane, enseignant à l'INSEPS). Ce témoignage corrobore avec celui du responsable de la lutte simple « Si vous enlevez les pratiques culturelles et magico-religieuses, ça ne sera plus de la lutte. C'est ça qui fait la différence avec tout ce qui est lutte olympique, lutte gréco-romaine, etc. Et ça, c'est pour nous, il faut que ça reste. Et ce sont des choses, je vous dis, ça existe. Il ne faut pas que les Africains se voilent la face et disent que ce sont des histoires. Celui qui veut être un grand lutteur dans ce pays sans intégrer les pratiques mystiques, ne sera jamais un grand champion. Maintenant, il y a beaucoup de charlatans, ça c'est différent. L'Afrique a ses mystères que les gens apprendront à connaître, comme l'Égypte a ses mystères » (entretien avec le responsable de la lutte traditionnelle sans frappe).

Nous constatons donc que la lutte sénégalaise contrairement aux autres activités sportives, accorde une place prépondérante aux pratiques magico religieuses. S'intéresser à la lutte, c'est d'une part tenir compte des manifestations culturelles qui lui donnent toute son identité, et d'autre part, réintroduire les rites et le sacré dans les nouveaux modes d'entrée dans l'activité.

\section{Discussion}

Les réponses fournies par les acteurs scolaires et les propos exprimés par les acteurs extrascolaires se rejoignent. Ils pensent tous que la lutte est le sport national. Elle est devenue de nos jours un spectacle comportant des enjeux sociaux (promotion sociale), politiques (utilisation $d u$ modèle $d u$ champion par les hommes politiques), économiques (tourisme, profession...) et éducatifs (donner et partager dans le rude respect de la hiérarchie, sacrifice corporel, ...) pouvant offrir de réelles opportunités.

Les résultats obtenus mettent en évidence trois composantes de représentation qui seraient plus particulièrement significatives des enjeux de la lutte. Les composantes "pratiques magico religieuses", "violence liée à l'usage de la frappe (aspects techniques) " et « économiques » organisent les représentations des acteurs interrogés. 


\section{1. Composante " pratiques magico religieuses"} et extrascolaires. Ils apparaissent comme un élément central de leurs représentations. Cette composante peut être assimilée au noyau central de la représentation défini au plan théorique par Abric et Flament (1994) comme un aspect non négociable. La présence des pratiques magico-religieuses et rituelles fait émerger une dimension attractive chez les amateurs et donnent à la discipline un caractère symbolique socialement partagé. La mise en scène des combats est tout à fait spectaculaire. Elle prend en compte des données fondamentales de la culturalité africaine comme les rituels magiques. Le combat ne se réduit pas à l'opposition entre deux poids lourds. L'avant match est l'occasion d'un interminable balai de rituels magico religieux compris dans la durée de la cérémonie. En effet, le lutteur et son entourage se voient dans l'obligation de se soumettre à des pratiques mystiques à des fins de protection, mais aussi pour se rassurer et se motiver. Chaque lutteur fait appel à un ou plusieurs marabouts. Ils constituent pour certains lutteurs la première force. Pour d'autres, c'est la deuxième force après les qualités physiques et techniques du lutteur.

Le marabout joue un rôle très important dans la vie du lutteur et dans le déroulement de ses combats. C'est lui qui décide de la place à occuper sur l'aire, de la manière de s'orienter, de la manière et du moment d'entrée dans le stade. Il peut même influencer l'heure du combat. Il donne au lutteur des amulettes, de l'eau bénite et il prédit souvent l'issue du combat. Il est assimilé à un préparateur mental. Ces rituels magiques, qui traversent la vie sociale sénégalaise de part en part ne sauraient concerner que cette pratique. Ces pratiques sont présentes aussi bien dans les secteurs encore fortement marqués par les pratiques natives comme les mariages, les baptêmes, les deuils et autres circoncisions, que dans les activités sociales liées à la modernité comme le travail à l'usine, la politique, etc. Nous pouvons alors comprendre que la non prise en compte de ces éléments par les enseignants d'EPS peut expliquer l'absence d'engouement vis à vis de la lutte scolaire.

\section{2. Composante « violence»}

Certains élèves et certains enseignants déplorent la violence des combats liée à l'utilisation abusive de la frappe qui risque de dénaturer l'activité. Pour eux, la frappe doit permettre une ouverture pour enchaîner sur une prise. Ainsi la composante "violence» semble être un apport de connaissance non négligeable pour l'intégration de la lutte à l'école. En effet, l'activité en elle-même n'a jamais été prise en compte dans la formation, dans la théorisation ou bien dans la définition de contenus de l'éducation physique. C'est une pratique qui jusqu'ici se menait hors de l'école. Et son entrée dans l'école nécessite un certain nombre de traitements pour supposer l'utilisation de la frappe à l'école.

Il faut aussi noter que dans le contexte de la société sénégalaise, la lutte est réservée aux hommes. Les filles ne luttent pas souvent, elles se contentent de chanter, de danser, etc. Se pose ici le problème de la mixité à l'école. Est-ce qu'il faudrait envisager cet enseignement uniquement pour les garçons? À quelles conditions, les filles en rapport avec les modèles en vigueur pourront adhérer à cette forme d'activité ? Il faut donc tenir compte des représentations d'ensemble qui affectent non seulement les

eJRIEPS, 20 | 2010 
enseignants, qui sont aussi les produits d'un milieu bien déterminé, les parents d'élèves, les élèves proprement dit et l'institution scolaire.

\section{3. Composante « économique»}

Cette composante est assez majoritaire dans les réponses des lutteurs, des promoteurs et des managers. Elle est aussi présente dans les réponses des élèves et des enseignants. Avec l'évolution actuelle, on note des changements dans la représentation qu'on se fait de la lutte. Durant les années 50-70, les lutteurs étaient marginalisés. La lutte était l'apanage des ruraux, des couches populaires. Aujourd'hui, avec l'importance des cachets, la lutte touche presque toutes les couches de la population sénégalaise. Les fédérations sont dirigées par des magistrats, des médecins, des professeurs d'université, etc. Les combats sont souvent parrainés par les hommes politiques (président de la république, premier ministre, etc.). Le lutteur offre aussi une image valorisante à travers son profil actuel (professionnel). On peut alors noter une évolution dans la représentation que la société se fait du lutteur.

Un combat de lutte n'est pas simplement un affrontement physique. Il y a des enjeux économiques, des chants, des danses, des rythmes, qui sont spécifiques à la lutte, faisant du lutteur un représentant de sa communauté. Les backs représentent la poésie. La danse, les chants, la tenue et les techniques marquent la différence entre les ethnies. Pour être enseignée, il est nécessaire de faire un traitement didactique de la lutte.

\section{Conclusion}

Au Sénégal, comme dans la plupart des pays en voie de développement, le maintien des traditions s'avère de plus en plus difficile, dans un contexte international dominé par les concepts de progrès, de mondialisation et de globalisation. La lutte sénégalaise, activité corporelle et ludique, qui autrefois occupait une place importante dans l'éducation, la socialisation des jeunes, ne pouvait rester en marge d'une dynamique de transformation et d'évolution socioéconomique et professionnelle, voire symbolique, qui la touche directement. Elle est devenue de nos jours, une activité urbaine, un spectacle comportant des enjeux sociaux (promotion sociale), politiques (utilisation du modèle du champion par les hommes politiques), économiques (tourisme, profession...) et éducatifs (donner et partager dans le rude respect de la hiérarchie, sacrifice corporel, ...) pouvant offrir de réelles opportunités. Cette étude vise à identifier les éléments significatifs de représentations de la lutte sénégalaise. L'enjeu est de déterminer une forme de lutte scolaire.

L'analyse de l'activité montre qu'elle répond parfaitement aux objectifs qui sont dévolus à l'EPS. Cependant, la lutte scolaire doit faire face à deux obstacles majeurs. Il s'agit d'une part de l'ensemble des représentations qui entourent cette activité et d'autre part de la démarche à mettre en œuvre surtout par rapport aux compétences à développer. En effet, ce qui est paradoxal, c'est qu'à l'Institut National Supérieur Éducation Physique et Sportive de Dakar, les enseignants apprennent plutôt la lutte olympique, la lutte gréco-romaine et non la lutte sénégalaise. Cela veut dire qu'il n'y a pas de conception de la lutte traditionnelle en tant que support de l'enseignement. La production du savoir nécessaire et l'enseignement de ce savoir aux différents acteurs qui en auront la charge. En effet pour quelqu'un qui n'est pas préparé, la 
gestion de la classe en rapport avec les effectifs pléthoriques et la maîtrise d'une activité qui peut avoir des débordements du point de vue de la violence, peuvent être délicat. Ainsi, notre modèle d'élaboration des contenus d'enseignement de la lutte en milieux scolaires tient compte des contraintes institutionnelles de l'EPS (manque de moyens, effectifs pléthoriques, finalités éducatives, etc.), des savoirs et pratiques sociales de référence des luttes (les différents rôles sociaux, les pratiques rituelles, les aspects techniques et tactiques, etc.) et l'analyse des sports de combat de percussion et de préhension (boxe, judo, etc.).

À travers la pratique de la lutte, nous croyons déceler une diffusion de ces valeurs, qui faciliterait une meilleure intégration des citoyens sénégalais par le biais d'un "sport » authentique et moderne vraiment de «chez nous»(autochtone). L'analyse des résultats nous a permis de découvrir la structuration des représentations et les compétences présentes, utilisées dans l'activité. Ces compétences nécessitent d'être dépersonnalisées et organisées dans une logique opératoire pouvant se matérialiser dans une représentation transmissible.

\section{BIBLIOGRAPHIE}

Abric, J-C. (1987). Coopération, compétition et représentations sociales. Fribourg : Eds Del Val.

Abric, J-C. (1994). Pratiques sociales et représentations. Paris : PUF.

Abric, J-C. (1999). Psychologie de la communication. Paris : Ed. A. Colin.

Astolfi, J-P. \& Develay, M. (1989). La didactique des sciences. Paris : PUF.

Astolfi, J-P. (1992). L'école pour apprendre. Paris : ESF.

Aubert, J. (1997). Représentations sociales et comportements en gymnastique d'élèves de collège. Thèse de Doctorat, Spécialité Activités Physiques et Sportives, Université Grenoble 1.

Bardin, L. (1993). L'analyse de contenu. Paris : PUF.

Beart (1960). Recherches des éléments d'une sociologie des peuples africains à partir de leurs jeux. Paris : Présence Africaine.

Bourdieu, P. (1984). Questions de sociologie. Paris : Éditions de Minuit.

Bouthier, D. (1993). L'approche technologique en STAPS : représentations et actions en didactique des APS. Diplôme d'habilitation à diriger des recherches, Université Paris-Sud.

Capodi, M, (1994). Des représentations... partout ? In G. Bui-Xuân \& J. Gleyse (Eds), Enseigner L'EPS (pp. 99-110). Clermont-Ferrand : AFRAPS.

Clément, J-P. \& Lacaze, L. (1985). « Contribution à l'histoire sociale de la lutte en France. » Travaux et recherches en EPS $n^{\circ} 8$.

Cornu, L., \& Vergnioux, A. (1992). La didactique en question. Paris : CNDP.

David, B (1996). « Statut didactique des représentations dans l'enseignement du rugby », Impulsions, 2 . 
Devalay, M. (1992). De l'apprentissage à l'enseignement. Paris : ESF

Doise, W., \& Palmonari, A (1986). L'étude des représentations sociales. Neuchâtel, Paris : Delachaux \& Niestlé.

Dorville, C. (1991). « Représentations de l'enseignant par les parents d'élèves et les professeurs. » STAPS, 12(24).

During, B. (1984). Des jeux aux sports, deuxième partie. Paris : Vigot.

Fédération Française de Lutte. (1981). « Lutte, un programme d'apprentissage. » Revue EP.S, 171

Flament, C. (1989). Structure et dynamique des représentations sociales, In D. Jodelet (Ed) : Les représentations sociales (pp. 204-219). Paris : PUF.

Flament, C. (1994). « Aspects périphériques des représentations sociales. » In C. Guimelli (Ed), Structures et transformations des représentations sociales. Neuchâtel : Delachaut et Niestlé.

Giordan, A. \& De Vecchi, G. (1987). Les origines du savoir. Genève : Delachaux et Niestlé.

Guimelli, C. (1996). Valence et structure des représentations sociales. « Bulletin de psychologie », XLIX, 58-72.

Hebrard, A. (1986). L'Éducation Physique et Sportive, réflexions et perspectives. Paris : Revue EPS.

Irlinger, P., Louveau, C., \& Metoudi, M. (1992). « Sociologie des activités physiques et sportives : promenade dans la littérature. » Revue STAPS, 13(27).

Jodelet, D. (1989). Les représentations sociales. Paris : PUF.

Jodelet, D. (1999). Représentations sociales : un domaine en expansion, In D. Jodelet (Dir.), Les représentations sociales (pp. 47-78), 6 éd. Paris : PUF.

Kalalobe, I. (1962). « La vocation africaine du sport. » Présence Africaine, XLI, $2^{\mathrm{e}}$ trimestre.

Ly, O. (1996). De la dépréciation de nos activités sportives traditionnelles : exemples de la lutte sénégalaise. Mémoire de maîtrise STAPS, INSEPS, Dakar.

Mannoni, P. (1998). Les représentations sociales, Paris, PUF.

Marsenach, J. \& Merand, R. (1987). L'évaluation formative en EPS dans les collèges, Rapports de recherches $\mathrm{n}^{\circ} 2$, INRP.

Marsenach, J et al. (1991). EPS, Quel enseignement? Paris : INRP, Didactique des disciplines.

Marsenach J., \& Amade-Escot, C. (1993). Orientations de la recherche en didactique de l'EPS. Revue Française de Pédagogie, 103, 33-42.

Petrov, R. (1984). Lutte libre et gréco-romaine. Lausanne : Ed Fila.

Rouquette, M-L, (1994). Sur la connaissance des masses. Grenoble : PUG.

N'diaye, A-R. (1996). De la lutte traditionnelle chez les sérères fondement mythique, techniques et langages gestuels. Éd. Sépia.

Seca, J-M. (2002). Les représentations sociales. Paris : Armand Colin.

Terrisse, A. (1994). La question du savoir dans la didactique des APS. Habilitation à diriger des recherches. Université Paul Sabatier. Toulouse. 


\section{RÉSUMÉS}

À la fois pratique sportive et artistique, la lutte est un phénomène immense au Sénégal. Elle s'inscrit aujourd'hui dans le processus de passage des jeux aux sports selon le phénomène de sportivisation des jeux traditionnels, malgré son ancrage dans l'imaginaire sénégalais, lié au monde des croyances et des superstitions. Cette étude vise à approcher l'étayage des représentations des élèves, des enseignants, et des acteurs extrascolaires. Il s'agit de faire ressortir les points saillants des représentations sociales de la lutte sénégalaise, d'en étudier leur nature et d'en estimer l'importance, afin d'envisager leur utilité dans la construction d'une forme de lutte scolaire en EPS et le choix des modes d'entrées dans l'activité. Le croisement des réponses fournies par les enseignants, les élèves et les acteurs extrascolaires permet de découvrir la structuration des représentations utilisées dans l'activité et il renseigne sur la culture de l'activité qu'il conviendrait de transmettre à l'élève en EPS. 